\title{
Interaction modes between nanosized graphene flakes and liposomes: Adsorption, insertion and membrane fusion
}

\author{
Raul Santiago, Ramon Reigada* \\ Department de Ciència dels Materials i Química Física and Institut de Química Teòrica i Computacional (IQTCUB), Universitat de Barcelona, Barcelona, Spain
}

\section{A R T I C LE INFO}

\section{Keywords:}

Graphene

Graphene oxide

Liposome

Coarse-grained molecular dynamics

Computer simulations

Membrane fusion

\begin{abstract}
A B S T R A C T
Background: Understanding the effects of graphene-based nanomaterials on lipid membranes is critical to determine their environmental impact and their efficiency in the biomedical context. Graphene has been reported to favourably interact with biological and model lipid membranes.

Methods: We report on a systematic coarse-grained molecular dynamics study of the interaction modes of graphene nanometric flakes with POPC/cholesterol liposome membranes. We have simulated graphene layers with a variety of sizes and oxidation degrees, and we have analyzed the trajectories, the interaction modes, and the energetics of the observed phenomena.

Results: Three interaction modes are reported. Graphene can be transiently adsorbed onto the liposome membrane and/or inserted in its hydrophobic region. Inserted nanosheets prefer a perpendicular orientation, and tilt in order to maximize the contact with phospholipid tails while avoiding the contact with cholesterol molecules. When placed between two liposomes, graphene facilitates their fusion in a single vesicle.

Conclusions: Graphene can be temporary adsorbed on the liposome before insertion. Bilayer curvature has an influence on the orientation of inserted graphene particles. Cholesterol molecules are depleted from the surrounding of graphene particles. Graphene layers may catalyse membrane fusion by bypassing the energy barrier required in stalk formation.

General significance: Nanometric graphene layers can be adsorbed/inserted in lipid-based membranes in different manners and affect the cholesterol distribution in the membrane, implying important consequences on the structure and functionality of biological cell membranes, and on the bioaccumulation of graphene in living organisms. The graphene-mediated mechanism opens new possibilities for vesicle fusion in the experimental context.
\end{abstract}

\section{Introduction}

Carbon-based nanomaterials have attracted extensive attention in recent years, and in particular, graphene, which possesses a number of extraordinary electronic, optical, mechanical and thermal properties $[1,2]$. Since the production of graphene and its derivatives is rapidly growing [3] and considering that they will be released into the environment at some points of their life cycle, their toxic effects and environmental impact are matters of serious concern [4]. Moreover, graphene oxides (i.e. graphene functionalized with hydroxyl, epoxy and carboxyl groups that render it suitable to interface with biological systems) are now expanding its territory towards biomedical applications such as drug delivery, implantable electronic devices, antibacter-

\footnotetext{
* Corresponding author.

Email address: reigada@ub.edu (R. Reigada)
}

ial materials, biological imaging and sensing, as well as the development of new biosensing, diagnostic and therapeutic devices [5-9].

Understanding the effects of graphene-based materials with biological entities is fundamental for the development of their biomedical applications as well as to the determination of their exposure risks [10-13]. It is necessary then to develop empirical and modeling tools to assess the effects of these compounds when they come in contact with biological cells, and in this context, the interaction with biological lipid-based membranes is fundamental. A better understanding of the physical principles governing these complex interactions can be achieved by using simple and reproducible systems, such as model lipid membranes. Several experimental studies have been recently performed on the effects of graphene-based materials acting on simple solid supported lipid membranes [14,15], unilamellar lipid vesicles 
$[15,16]$ and liposomes $[16,17]$. The affinity of graphene and graphene oxides for the hydrophobic region of the lipid membranes gives rise to a variety of modes of interaction that include the formation of multilayered graphene/membrane structures, the generation of pores in vesicle membranes, the insertion of graphene into liposomes or the complete destruction of the bilayer configuration [14-17].

Molecular dynamics (MD) simulations provide an excellent approach to unveil the ultimate molecular mechanisms regulating the behavior of interacting systems and they have been successfully used to obtain direct insights into many lipid membrane processes. Since the completion of the different graphene/membrane interaction modes may require hundreds of nanoseconds or up to several microseconds, coarse-grained (CG) MD simulations have been used to capture them, still preserving the molecular nature of the simulated species [18-21]. The simulations reveal that despite the intrinsic energy barrier against bilayer penetration, small graphene flakes may spontaneously enter a lipid bilayer by locally piercing it by a corner of the graphene sheet $[18,19]$. Local piercing by sharp graphene corners initiates membrane propagation along extended graphene edge until nanosheets become embedded in the bilayer $[19,20]$. A more destructive action mode is found for large graphene sheets, which can lead to the total bilayer disruption and the formation of stable lipid monolayers on the surface of graphene [21].

To date, molecular simulations addressing the interaction of graphene materials and lipid membranes are limited to planar lipid bilayers. However, biological membranes (plasmatic cell membranes and the membranes that shape the internal cell organelles) are not flat and may locally adopt significantly large curvatures due to their functionality and/or as a result of the interaction with other cell components (proteins, cytoskeleton, etc.) [22,23]. Therefore, a systematic study carried out with molecular-detailed approaches for the elucidation of the effects of membrane curvature on its interaction with graphene nanosheets is required, and this is the motivation of the present work. Moreover, biological membranes usually contain significant amounts of cholesterol (Chol), and this component is generally absent in previous MD simulations devoted to investigate graphene/membrane interaction modes. Cholesterol determines most of the physical properties of biomembranes; for instance, its tetracyclic ring system increases membrane packing, contributing to the fluidity of the membranes and reducing its permeability to specific molecules [24]. Therefore, in order to approach real biomembranes, vesicle membranes composed by a phospholipid and containing a significant fraction of cholesterol have been simulated in this work.

We have systematically investigated by means of CG MD the action modes of graphene nanosheets on a simple $30 \mathrm{~mol} \%$ cholesterol-containing lipid membrane forming a small liposome. Different graphene sizes, oxidation degrees and interaction orientations are analyzed in our simulations. We focus on different interaction modes and we pay special attention to the insertion process and the different manners that graphene flakes can be accommodated inside the liposome membrane, depending on their size and oxidation degree. Interestingly, the favourable interaction between graphene and the interior of the vesicle lipid membranes provides a novel graphene-mediated mechanism for vesicle fusion that is described for the first time by using MD simulations and opens new possibilities for vesicle fusion in the experimental context.

\section{Methods}

\subsection{Coarse-grained description}

The simulated molecules have been described according to the 4-to-1 coarse-graining implemented in the Martini v2.0 force field [25]. Beads forming a given molecule interact via bond distance, and bond and dihedral angle potentials that reflect the equilibrium properties of the molecule. The approach considers different types of interactive beads with particular degrees of polarity, charges and hydrogen bonding capabilities that are fixed according to an extensive calibration of the chemical building blocks of the CG force field against thermodynamic data (see Ref. [25] for details). A large variety of lipid membrane phenomena have been successfully investigated using the Martini force field [26], in some cases also by involving the interaction with carbon-based nanomaterials [27].

In our simulations the following molecular species have been simulated (see Fig. 1a). CG water particles are represented by highly polar particles, each one mapping 4 real water molecules. The phospholipid 1-Palmitoyl-2-oleoylphosphatidylcholine (POPC) is formed by 13 interacting beads representing: a positively charged choline group, a negatively charged phosphate, two neutral glycerols, and two tails with four and five apolar alkane-like particles, respectively. The third bead of the longer acyl chain accounts for the unsaturation in the methylene sequence for the oleoyl tail. Cholesterol is formed by eight particles: a polar bead for the hydroxyl group, five representing the ring sterol system and two for the short alkyl tail.

The CG parameterization of graphene and graphene oxide flakes follows the proposal described in Ref. [28] that reproduces quite well many structural and mechanical graphene properties. Graphene is modeled by means of a hexagonal lattice of apolar particles, each one representing four carbon atoms according to the 4-to-1 Martini rule. Although the Martini force field recommends a 3-to-1 mapping for the description of molecules with rings (such as cholesterol), here we choose the 4-to-1 coarse-graining in order to preserve the original hexagonal lattice symmetry of graphene as it has been used in [28] and in other simulations of this material with amphiphilic molecules [29].
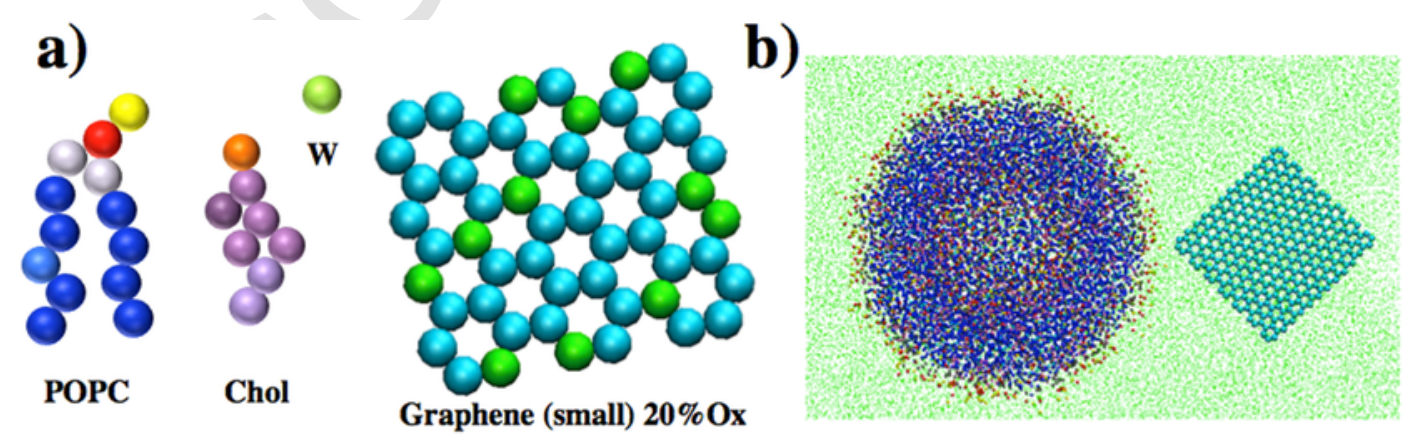

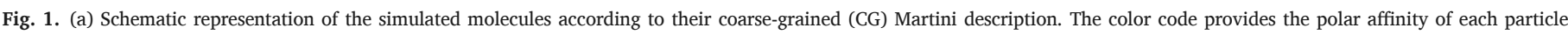

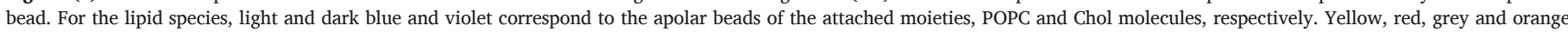

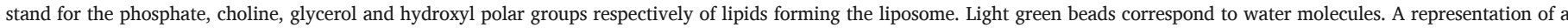

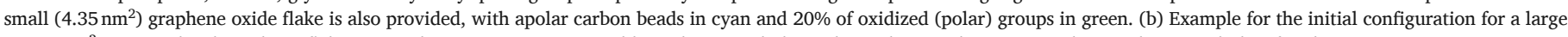
$\left(32.59 \mathrm{~nm}^{2}\right)$ non-oxidized graphene flake approaching its corner to a equilibrated POPC/Chol vesicle. In this panel water particles are shown with dots for clarity. 
Other carbon-based nanomaterials like nanotubes [30] and fullerenes [31] have been also successfully modeled following a 4-to-1 approach. The equilibrium distance between connected beads is set to $0.28 \mathrm{~nm}$, twice the carbon-carbon distance in atomic graphene. Most of the graphene force field parameter values optimized in Ref. [28], such as the spring constant for the dihedral interaction and the Lennard-Jones parameters that account for non-bonded interactions, are used in our simulations. Instead, bond distances and angles are fixed as constraints in the simulated dynamics because the values assigned to the force constants for the bond distance and angle are so large [28] that they would force extremely small time steps, making simulations impractical. In the limit where the graphene axial stiffness is much larger than the membrane in-plane stiffness, this approximation is rather inconsequential; namely, no significant in-plane deformations of graphene are expected due to its interaction with a membrane (soft-matter) system.

Different graphene layers are used in our simulations by varying their size and oxidation degree. Size is varied from very small flakes of 54 beads (linear size slightly smaller than membrane thickness and surface $\approx 4.35 \mathrm{~nm}^{2}$ ) to large layers made of 350 beads (linear size slightly larger than the liposome radius and surface $\approx 32.6 \mathrm{~nm}^{2}$ ). Intermediate sizes (medium-small: 120 beads and surface $\approx 10.5 \mathrm{~nm}^{2}$, and medium-large: 190 beads and surface $\approx 17.1 \mathrm{~nm}^{2}$ ) are sometimes used in the simulations. The oxidation degree is varied from $0 \%$ (all graphene beads are apolar) to $20 \%$ (one in five beads are randomly given a polar character). Occasionally, graphene $10 \%$ and $30 \%$ oxidized graphene flakes are also used in the simulations.

\subsection{Simulation protocols}

Molecular dynamics simulations have been performed using the GROMACS v4.5.5 software package [32]. The simulations are carried out in the NpT ensemble through a weak coupling algorithm at $T=310 \mathrm{~K}$ and an isotropic $p=1 \mathrm{~atm}$. Electrostatic interactions are handled using a shifted Coulombic potential energy form and charges are screened with a relative dielectric constant $\varepsilon_{\mathrm{r}}=15$. Non-bonded interactions are cut off at $1.2 \mathrm{~nm}$. Periodic boundary conditions are used in all three directions, and the time step is set to $20 \mathrm{fs}$. It is important to notice that the interpretation of the time scale in Martini simulations is not direct: the energy landscape is significantly "smoothed" due to the coarse-grained potentials respect to atomistic approaches, so that the effective time scale is larger than the actual simulation time. Often, a conversion factor of 4 is used, since it is the speed-up factor needed to capture the correct diffusional dynamics of CG water particles compared with real atomistic water molecules [25]. Here, however, the original simulation time units are preserved.

The simulated liposome is built with 614 POPC and 263 Chol molecules ( $30 \mathrm{~mol} \%$ of Chol) forming a closed bilayer structure. The inclusion of Chol provides the membrane liposome with in-plane fluidity and flexibility properties similar to biological lipid membranes. The liposome is hydrated with 71,552 water particles (15\% of antifreeze water particles has been used to prevent anomalous water freezing [25]), and conveniently equilibrated during $1 \mu \mathrm{s}$ at $310 \mathrm{~K}$ and $1 \mathrm{~atm}$. At equilibrium the vesicle diameter is about $10 \mathrm{~nm}$, and the water density inside and outside the liposome becomes stabilized around 980 g/L. 427 POPC molecules form the outer leaflet and 187 POPC molecules form the inner leaflet. Chol molecules flip-flop frequently between the two leaflets, the inner layer being more concentrated with Chol (35-40 mol\%). Both leaflets display a fluid behavior, and no holes/pores are observed [33].

The simulated systems consist in a graphene nanosized flake that is placed in the aqueous phase at a distance of a few nanometers of the equilibrated liposome (see for instance Fig. 1b). We vary the flake size and its oxidation degree, and for each case, different initial graphene orientations are tried by placing one of its faces, edges or corners prox- imal to the liposome. The graphene/liposome systems are then subjected to an energy minimization process in order to eliminate possible particle overlaps, and production runs of $1 \mu \mathrm{s}$ are finally performed for all cases. Additional simulations are performed starting from graphene-inserted configurations and modifying the oxidation degree of graphene in order to observe the effects on graphene's accommodation inside the vesicle bilayer.

In order to asses the effects of curvature in the behavior of inserted graphene layers, simulations of planar membranes have been also performed for comparative purposes. The simulated planar membranes consist of a mixture of 576 POPC molecules and 248 Chol molecules (30 mol\%) equally distributed at both leaflets. The membranes are conveniently hydrated with 16,000 water particles and equilibrated for $1 \mu \mathrm{s}$ in the NpT ensemble at $T=310 \mathrm{~K}$ and using an anisotropic $p=1 \mathrm{~atm}$. Graphene layers of different sizes are then placed between the two layers of the membranes and its behavior is tracked for $4 \mu \mathrm{s}$ of simulation.

Simulation trajectories are examined by some of the analysis tools included in the GROMACS package and own developed applications. Molecular pictures presented in this paper have been performed using Visual Molecular Dynamics, VMD [34].

\section{Results and discussion}

\subsection{Interaction modes: adsorption and insertion}

As described in the previous studies based on MD simulations $[19,20]$, the affinity of graphene for the apolar part of the lipid membrane causes nanometric graphene flakes to interact favourably with the hydrophobic region of the bilayer. Although in most situations the flake penetrates the membrane, sometimes our simulations show a relatively stable mode of interaction that corresponds to an adsorption on the outer layer of the liposome bilayer. For example, all simulations performed with the smallest flakes display, independently of the initial arrangement and oxidation degree, a frontal adsorption by one of the flake faces on the outside of the vesicle. The details of this configuration are presented in Fig. 2a. The graphene sheet is adsorbed by contacting one of its faces with the outer lipid layer of the vesicle. The polar heads of the lipids of this leaflet move to the sides and their apolar chains are twisted and arranged tangentially to the liposome in order to accommodate the graphene layer and allow it to approach the center of the membrane (Fig. 2a). Interestingly, since cholesterol does not possess the flexibility of phospholipids, cholesterol molecules avoid being located in the outer leaflet of the bilayer just below the graphene layer. Larger flakes also show the adsorption mode; see Fig. $2 \mathrm{~b}$ for one of the large size simulated graphenes. In this case, phospholipds placed at the edges of adsorbed graphene twist and orient their apolar tails in order to cover it from below, whereas those placed in the central region flip, contacting graphene with their acyl chains and bringing their headgroups away from it. In all adsorption cases, the thickness of the bilayer is locally reduced in approximately a $20-30 \%$ at the adsorption region, and the remaining part of the liposome membrane preserves its original bilayer configuration (no pores or other alterations are observed).

Since graphene is more stabilized when fully inserted into the lipid membrane, the adsorption mode corresponds to a metastable (transient) configuration. This mode generally takes place when the graphene approach is frontal. For smaller flakes, even if graphene corner or edge is initially proximal to the liposome, the graphene quickly rotates and contacts frontally, resulting in the adsorption behavior described above. The stability of the adsorption mode depends on the size and oxidation degree of the graphene sheet. Intermediate and large graphene layers can remain absorbed for times longer than those achievable in MD simulations. For instance, the adsorption mode per- 

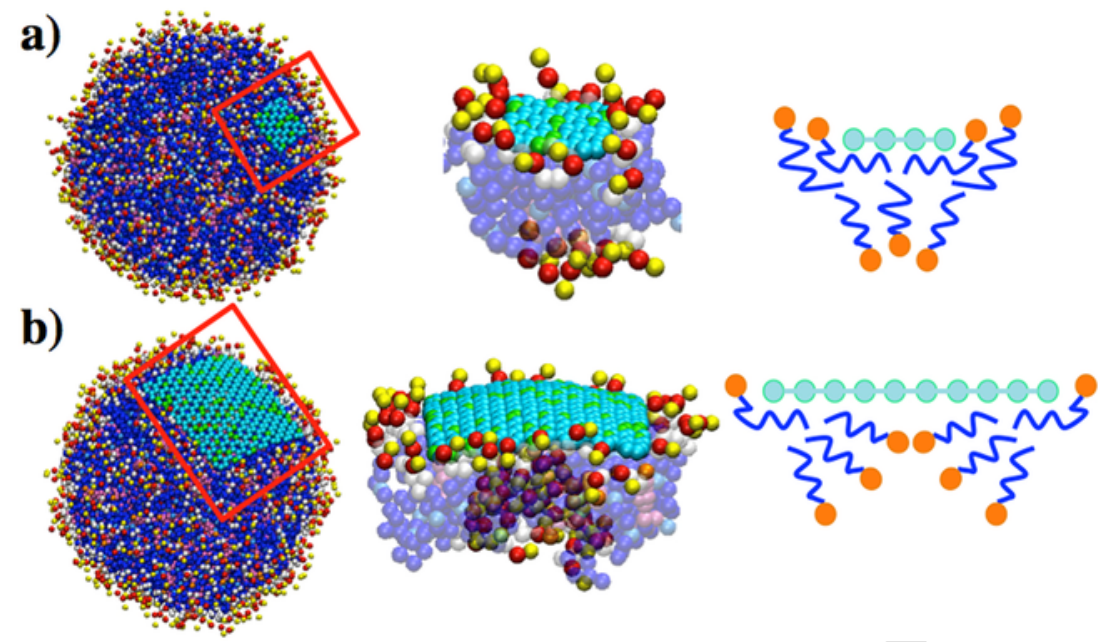

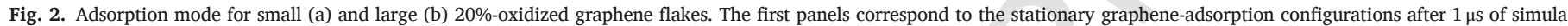

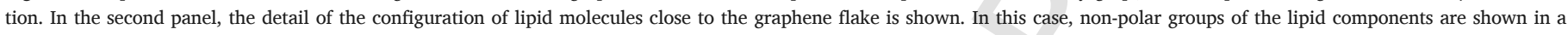

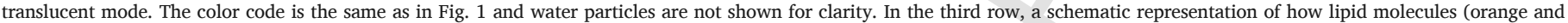
blue) accommodate the adsorbed graphene flake (cyan) is presented.

sists for the large graphene sheet shown in Fig. $2 \mathrm{~b}$ if the simulation is extended up to $5 \mu \mathrm{s}$. Adsorption of the small sized simulated graphene flakes, on the other hand, is much less stable: apolar graphene remains adsorbed only a few tens of nanoseconds, whereas $20 \%$-oxidized sheets get trapped in the adsorption mode for several microseconds (for example, the small-sized flake in Fig. 2a remains adsorbed before insertion for $2.3 \mu$ s of extended simulation).

Due to the apolar nature of graphene, full insertion into the liposome bilayer is the most stable interaction mode. As stated above, the simulations performed with the small flakes always show immediately a transitory adsorption stage. At some point (depending on its oxidation degree) the adsorbed layer is tilted and it begins to pierce the membrane due to the attractive interactions between the graphene and the tail groups of lipids. When this happens, the graphene sheet is rapidly pulled inside towards the hydrophobic core of the lipid bilayer and ends up embedded in the liposome membrane (see Fig. 3a). Simulations with intermediate and large sized graphene layers that approach one of their edges or corners to the liposome display spontaneous penetration (see Fig. 3b), although in these cases, the complete coverage of the graphene layer is achieved more slowly due to their larger size (small flakes are completely covered by lipids after a few nanoseconds whereas the largest sheets need more than one microsecond). As explained above, intermediate and large simulated graphene layers are better inserted inside the liposome when the approach is not attempted frontally, otherwise graphene can be trapped in an adsorption configuration at the outer part of the liposome for long times. The fact that graphene entry is initiated at corners or edges was already reported for planar membranes in previous CG MD simulations [18-20].

In this work, graphene layers of sizes ranging from the thickness of the lipid bilayer to the radius of the liposome have been considered. CG MD Martini simulations with larger graphene assemblies (greater than the liposome's diameter) result in nonsense artifacts due to the artificial freezing of the aqueous phase. The use of antifreeze water prevents the artificial freezing of the system for the simulations reported in this work, but it is not enough to do so in the case of graphene layers of a size of the order of the simulation box. It is expected that the action of large graphene layers may cause the destruction of the lipid bilayer configuration as reported in some previous works simulating graphene supports [21]. Therefore, the action on closed lipid bilayer structures could correspond to the collapse modes described in CG MD simulations of liposomes contacting hydrophobic solid surfaces (see Refs. [33,35,36] for more details).

\subsection{Configuration of inserted graphene layers}

Previous simulations with cholesterol-free and flat membranes report a parallel arrangement of the inserted graphene at the mid-plane of the membrane [18-20]. In these works, a combination of insertion and rotation is described until reaching the final horizontal position in the middle of the bilayer, whereas perpendicular or tilted configurations across the membrane are only found when two opposite sides of graphene are sufficiently oxidized $[19,20]$. In our simulations, however, we observe how the horizontal position (between the layers of the membrane) is generally avoided regardless of the size and degree of oxidation of the graphene flakes. For example, all simulated small graphene layers (oxidized or not) end up embedded in the membrane with a perpendicular orientation respect to the liposome bilayer plane (parallel to lipid molecules). Once inserted, intermediate and large sized graphene layers also avoid being located in the middle of the liposome membrane and show a tilted conformation across the bilayer in order to maximize its coverage inside the membrane.

In order to check whether this configuration is the most stable or a transient state, we have conducted simulations where non-oxidized graphene flakes are initially placed between the two layers of the liposome membrane. In all cases, graphene sheets rotate from the initial parallel orientation to adopt a perpendicular/tilted configuration. As an example, simple visual inspection of the simulation trajectory for a medium-small graphene layer in Fig. 4a shows a quick graphene rotation to the perpendicular/tilted position in the nanosecond scale. Analogous simulations for planar POPC $/ 30 \mathrm{~mol} \% \mathrm{Chol}$ membranes reveal that apolar graphene sheets remain at the bilayer mid-plane as it is reported in Refs. [18-20], with the only exception of the smallest-sized flakes that adopt a perpendicular orientation after $50 \mathrm{~ns}$. Larger flakes display the expected behavior and remain at the mid-plane after $4 \mu \mathrm{s}$ of simulation (see Fig. 4b for a medium-small graphene particle).

The graphene layer rotation is tracked by computing at different times its orientation angle, $\alpha$, as the angle between the radial vector connecting the center of masses of the liposome and graphene, and the normal vector of the graphene plane (circles in Fig. 4c). For planar membranes, the orientation angle corresponds to the angle between the graphene and membrane planes (squares in Fig. 4c). The results reported in Fig. 4c reveal the two finding commented above. First, very small graphene sheets prefer a perpendicular orientation even when inserted in planar membranes. Previous works [18-20] reported a clear 


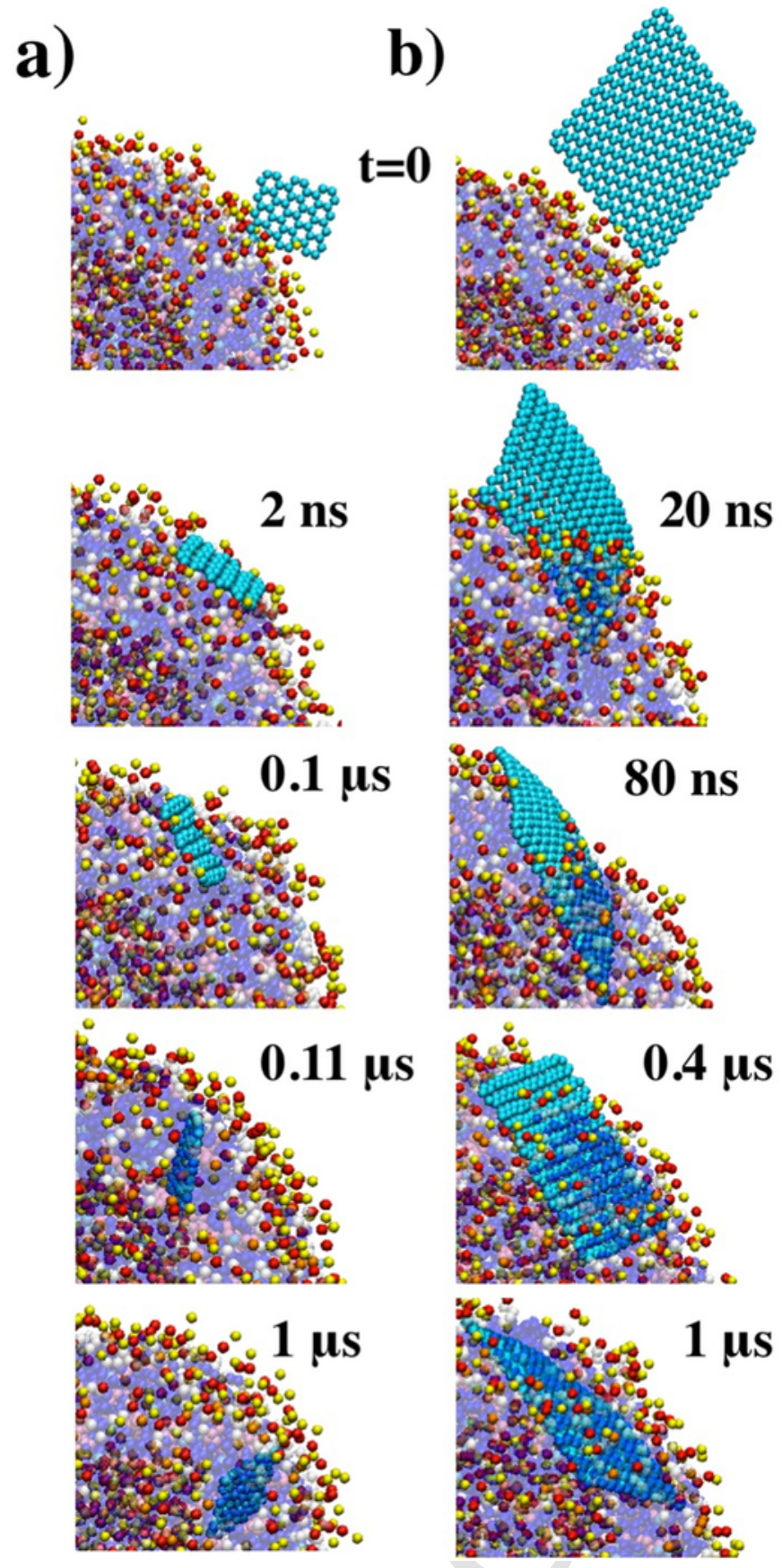

Fig. 3. Sequence of the insertion process for $0 \%$-oxidized small (a) and large (b) graphene flakes. Non-polar groups of the lipid components are shown in a translucent mode. The color code is the same as in Fig. 1, non-polar groups of the lipid components are shown in a translucent mode, and water particles are not shown for clarity.

preference to be sandwiched between the two layers of the bilayer but the simulated graphene sheets were in all cases larger than our smallest-sized layers. The behavior of our intermediate and large graphene layers confirm the preference to remain in the membrane mid-plane. Second, and more important in the context of this work, membrane curvature clearly favors a perpendicular/tilted position of inserted graphene sheets. When starting from a mid-plane position in the liposome bilayer, graphene rotation takes place in a few nanoseconds for the smallest sized flakes, and in several tens of nanoseconds for intermediate graphene sizes (Fig. 4c).
Some other interesting features can be also obtained by looking at the variations of the energetics of graphene when interacting with the other system components (see Fig. $4 \mathrm{~d}$ for the rotation process of the medium-small flake in Fig. 4a). The rotation from a parallel to a perpendicular/tilted orientation implies an increment of the number of favourable contacts between graphene and POPC molecules, together with a expected but small increase of contacts with water particles. Interestingly, the contacts with cholesterol molecules are markedly reduced, which implies a depletion of these molecules around the graphene flake once it is arranged perpendicularly. Summing up the variations of all the contributions, the total potential energy associated to graphene is slightly lower in the perpendicular/tilted position with respect to the parallel one (see black curve in Fig. 4d). Entropic considerations have to be also taken into account. Once the graphene layer adopts the perpendicular configuration it undergoes fast Brownian motion, including "lateral" diffusion along the membrane and rapid rotation. All these motion modes are clearly favoured when the graphene nanosheet stands parallel to the phospholipid molecules of the membrane so it can take advantage of their lateral flexibility. This rationale is also supported by the depletion of cholesterol molecules (much less flexible than POPC) around graphene. Moreover, graphene rotation modes are rather impeded when lying in the mid-plane since they imply local alterations of the membrane thickness that are energetically penalized.

Similar results than those presented in Fig. 4 for a completely apolar graphene are found for other small graphene flakes of different degrees of oxidation. The orientation of the graphene layer inside the liposome membrane is determined by its size, whereas the influence of its oxidation degree is less significant. We have systematically computed the graphene orientation angle for small, medium-small, medium-large and large sized graphene flakes with $0 \%, 10 \%, 20 \%$ and $30 \%$ of oxidation sites. The results are plotted in Fig. 5a for the simulations that start with the graphene corner proximal to the liposome membrane (as explained in Section 2.2), and the values of $\alpha$ correspond to the average over the last $0.25 \mu$ s of simulation when the graphene is already fully inserted inside the membrane. We obtain statistically indistinguishable results from simulations that start with the stable configuration of the apolar graphene layer and whose degree of oxidation is progressively increased. For each change of oxidation level, we conducted a simulation of $0.25 \mu$ s of equilibration followed by a production simulation of $0.25 \mu$ s with which the new value of the orientation angle has been calculated.

Small flakes have a linear size slightly smaller than the bilayer thickness so they are placed practically perpendicular to the membrane plane (Fig. 5a and b). Medium-small sheets are slightly larger than the thickness of the membrane so they display a small tilt with respect to the radial direction (Fig. 5a and c). Medium-large and large sized graphene layers are much larger than the bilayer width, thus they adopt quite inclined configurations (Fig. 5a and d-g). Interestingly, graphene orientation is not significantly affected by its level of oxidation (Fig. 5a). Increasing the oxidation level of the graphene sheet only implies a displacement to external positions for medium-large and large sized layers (compare Fig. 5d and e with Fig. $5 f$ and g).

The interaction and affinity of the inserted graphene flakes with the other components of the system has been also studied for the set of simulations reported above. With this purpose, the amount of molecules of POPC, Chol and water that are proximal to graphene have been measured from our simulations. We say that a molecule is proximal when any of its constituent simulation particles is closer than $0.5 \mathrm{~nm}$ to any graphene bead. As for the orientation angle, the values of the number of proximal molecules correspond to the average over the last $0.25 \mu$ s of simulation. The results are summarized in Fig. 6 and, as expected, they reveal a clear preference of graphene to be close to POPC molecules. Notice also that in all cases, considerable cholesterol 
a)
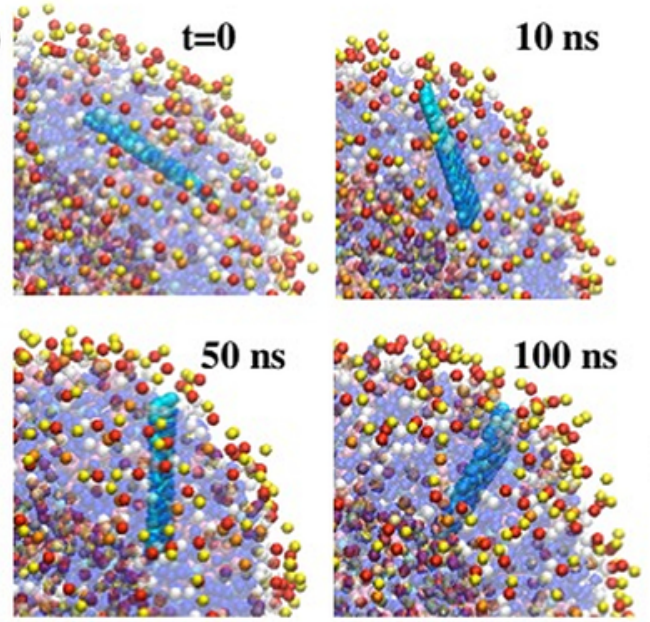

b)

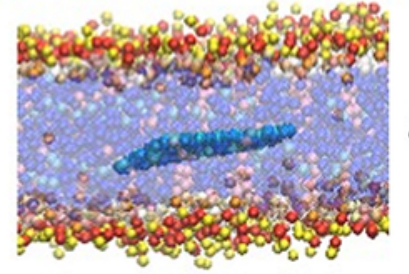

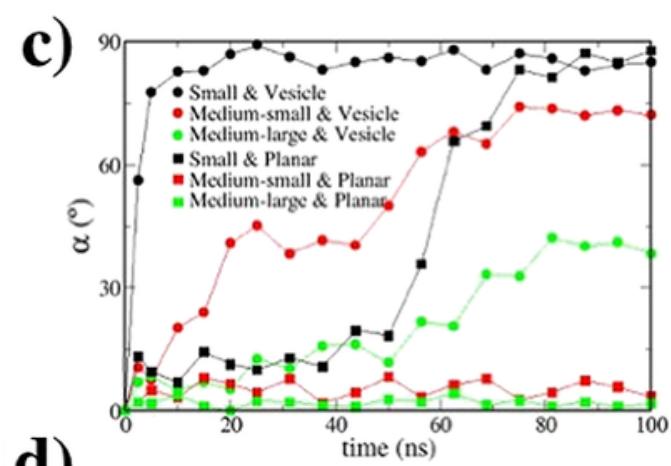

d)

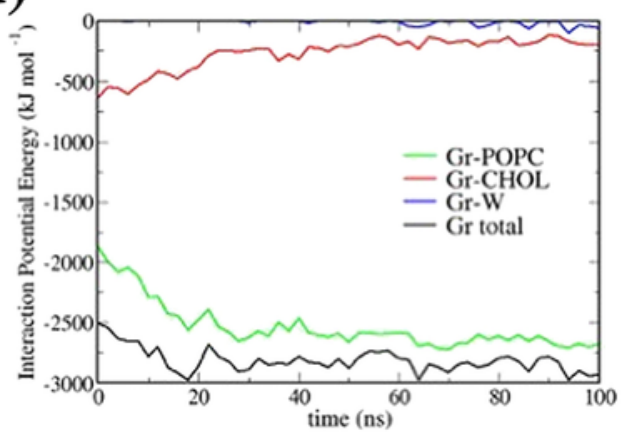

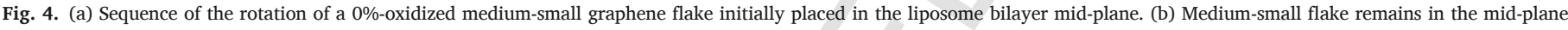

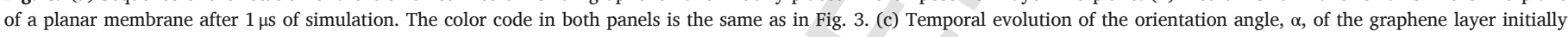

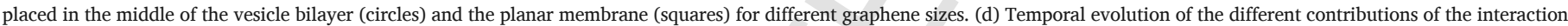
potential energy of graphene with other system components for the case in panel (a).

depletion around the inserted graphene layers is observed. The number of proximal POPC molecules is about 10 to 25 times larger than the number of proximal Chol particles, a proportion that is, in any case, much higher than the POPC/Chol ratio in the bilayer $(\approx 2.33)$. As suggested above, graphene motion and rotation is more favoured when surrounded with flexible POPC molecules than when close to rigid cholesterol particles. Finally, graphene-water contacts are extremely rare for the apolar graphene flakes, but they increase for the higher oxidized degrees (Fig. 6). This increase is particularly significant for medium-large and large layers with a linear size much greater than the membrane thickness, so that they partially slide off the membrane and establish favourable contacts of their polar sites with water molecules.

\subsection{Graphene-mediated vesicle fusion}

The insertion ability of nanometric graphene flakes can result in interesting effects. For instance, graphene-mediated vesicle fusion is observed when one of the simulated large-sized apolar graphene sheets is placed between two lipid vesicles (see Fig. 7a). The graphene flake is initially piercing both vesicles with two of its corners ( $\sim 1 \mathrm{~nm}$ deep). Graphene insertion into each vesicle results in an effective pulling force that brings the two vesicles together $(\sim 12.5 \mathrm{~ns})$. During the approach of the vesicles, the graphene layer strongly perturbs the surrounding lipid molecules and lipid extraction from the two vesicle bilayers is observed, forming a monolayer on both faces of the graphene sheet $(\sim 37.5 \mathrm{~ns})$. In a last stage, the graphene flake its absorbed $(\sim 200 \mathrm{~ns})$ and fusion is completed ( $\sim 75 \mathrm{~ns})$.

The variation of the different potential energy contributions during the fusion process provides a clear picture of its spontaneous nature due to graphene mediation (Fig. $7 \mathrm{~b}$ ). The fusion action involves a reduction of the water/lipid interface, namely, a strong reduction of unfavourable contacts between lipid and water molecules, and the consequent increment of water-water and lipid-lipid favourable interactions. At the same time, although not relevant for the total energy variation, graphene becomes more stable when absorbed in a lipid environment. Interestingly, the total potential energy variation only shows a tiny energy activation $\left(\sim 1 \mathrm{k}_{\mathrm{B}} \mathrm{T}\right)$ during the vesicle-vesicle approach, whereas no activation energy is required to complete the different fusion stages once the two vesicles contact ( $t \geq 12.5 \mathrm{~ns}$ in Fig. 7a).

It is important to notice that graphene is indispensable in the fusion phenomenon described in Fig. 7. In the absence of graphene, no fusion is observed in a simulation of two vesicles initially placed at a distance of $\sim 1 \mathrm{~nm}$. The vesicles display a random (diffusive) motion and several contacts are noticed in $10 \mu$ s of simulation, but no spontaneous fusion is observed. The graphene-mediated fusion mechanism requires, however, a particular orientation of the graphene particle; not all initial configurations lead to vesicle fusion. For example, a parallel orientation of the graphene layer respect to the two facing vesicles generally results in the adsorption of graphene onto the membrane of one of the liposomes and no fusion occurs. Instead, the corner approach favors the fusion process, in particular when the graphene pierces the two neighboring membrane vesicles to a similar extend (otherwise the graphene particle gets rapidly inserted in the closest liposome membrane and no fusion occurs). When this condition applies, the fusion takes place spontaneously and the process described in Fig. 7 is robustly reproduced.

Fusion of lipid bilayers in form of planar bilayers or vesicles has been largely studied from experimental, mathematical and numerical perspectives. It is generally assumed that fusion events follow the stalk-pore mechanism $[37,38]$ that is described in the three following stages. First, the two membranes contact at some point and a transient highly curved stalk quickly forms at the contact region. Immediately after, the stalk expands, the inner monolayers of fusing bilayers merge and a hemifusion diaphragm is formed. Finally, at some point, a fusion pore is formed, the bilayer ruptures and the fusion process is completed. Coarse-grained molecular dynamics $[39,40]$ and dissipative particle dynamics [41] simulations have captured the molecular details of the stalk model for bilayer fusion. The energetics of the fusion pathway 


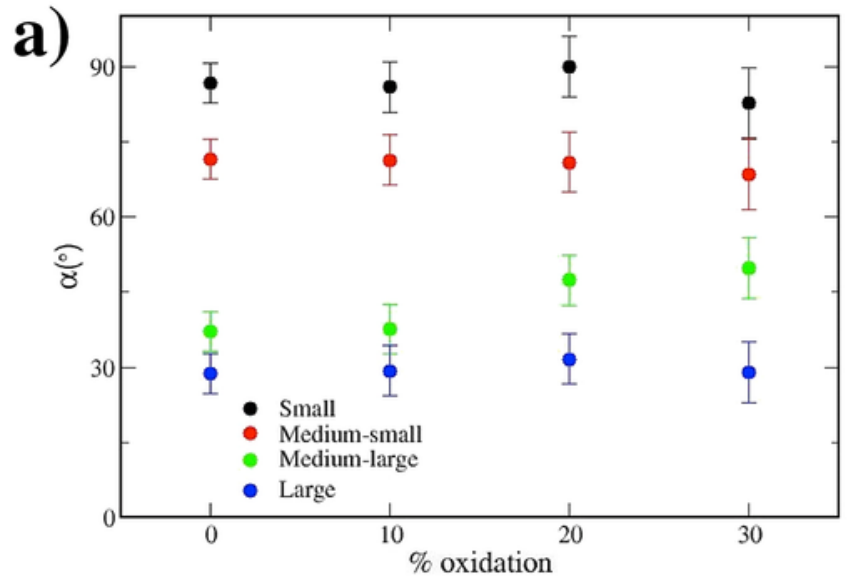

b)
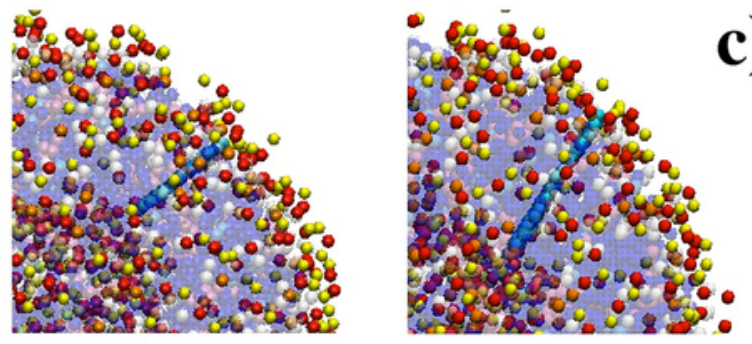

d)
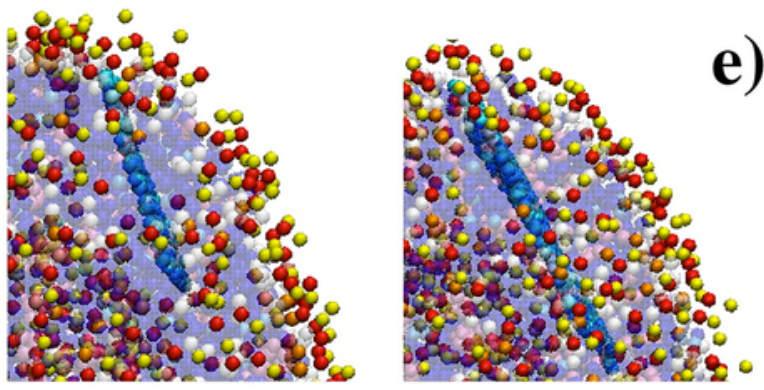

f)
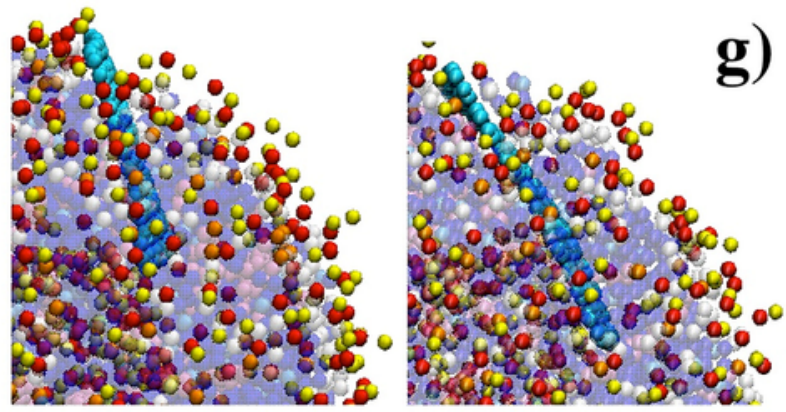

Fig. 5. (a) Graphene orientation angle, $\alpha$, for different particle sizes and oxidation degrees. The angle value corresponds to the average over the last $\mu$ s of simulation and the error bar stands for the standard deviation. (b-g) Graphene-liposome configuration after $4 \mu$ s of simulation for different graphene layer sizes and oxidation degrees: (b) small and apolar, (c) medium-small and apolar, (d) medium-large and apolar, (e) large and apolar, (f) medium-large and $30 \%$ oxidized and (g) large and $30 \%$ oxidized. The color code is the same as in Fig. 3.

generally involves a critical step with a high activation energy: the stalk formation. Stalk nucleation implies the local approach of the two membranes and a strong perturbation of the contacting lipids so that some lipid tails of opposing vesicles make contact at the polar bilayer-bilayer interphase as a previous stage to initiate the hydrophobic stalk domain [42]. Lipid disordering at the contact region and the protrusion of the lipid hydrophobic tails into the hydrophilic layer (or the opposite membrane) are highly demanding energy events. The activation energy of the whole fusion process has been estimated by different

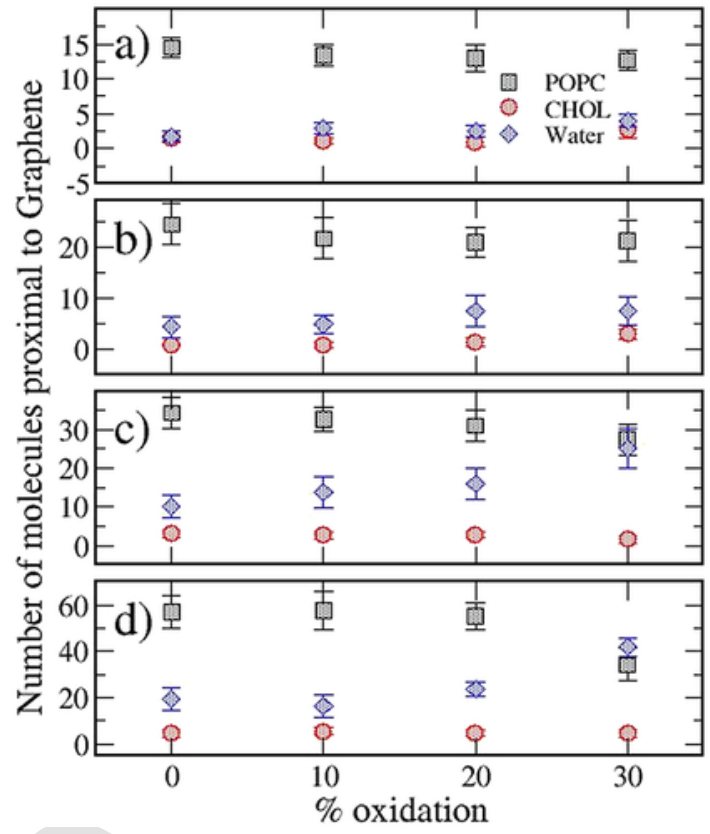

Fig. 6. Number of molecules (POPC, Chol and water) proximal to graphene for layers of different sizes and oxidation degrees. The value corresponds to the average over the last $\mu$ s of simulation and the error bar stands for the standard deviation. The different panels correspond to (a) small (b) medium-small, (c) medium-large and (d) large graphene layers.

mathematical and numerical approaches resulting in energy barriers of the order of several tens of $\mathrm{k}_{\mathrm{B}} \mathrm{T}$ energy units, depending on the lipid composition and membrane curvature [43-45]. The simulation described in Fig. 7 clearly reveals how lipid disordering and protrusion induced by graphene at the contact region practically eliminate the activation energy of the process, suggesting a mechanism for fusion catalysis mediated by graphene nanoflakes.

\section{Conclusions}

The analysis of simulations has unveiled the main molecular aspects that describe three interaction modes between graphene and liposomes: adsorption, insertion and fusion. They are summarized as follows. First, the adsorption of graphene layers has been captured and described. In this interaction mode, graphene contacts one of its faces to the outer layer of the liposome membrane, whose lipids locally splay and twist in order to provide a hydrophobic environment to the graphene contact surface. Interestingly, rigid cholesterol molecules avoid being placed in the contact region. Despite its transient nature, adsorption of layers with a linear size of the order of the liposome radius could remain longer times than several tens of microseconds; namely, period times that could be relevant in the biological context. The most favourable interaction mode, however, corresponds to the insertion of graphene into the liposome membrane. As it was already reported in previous simulation studies, spontaneous insertion is more favourably initiated at the corners or asperities of the graphene particle. Once inside, small graphene sheets with a linear size equal or smaller than the membrane thickness adopt a perpendicular orientation across the membrane. Increasing the graphene size results in the inclination of the inserted layer so that its coverage is maximized. Increasing the oxidation degree of the graphene particles makes them to partially slide off the membrane towards the aqueous phase. Comparison with simulations of flat membranes clearly indicates that large membrane curvatures hinder the preference of graphene sheets for being sandwiched between the two leaflets as it happens in completely planar membranes. According to our simulations, not only the 

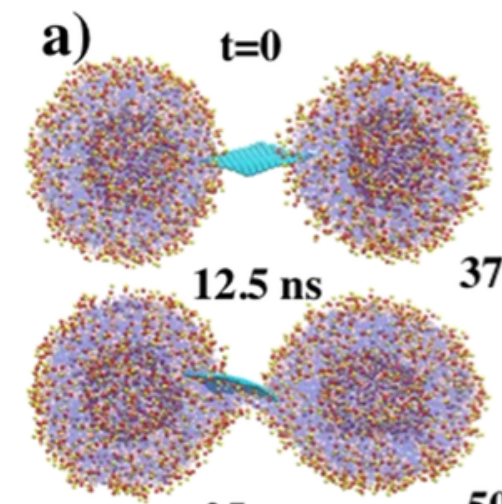

$37.5 \mathrm{~ns}$
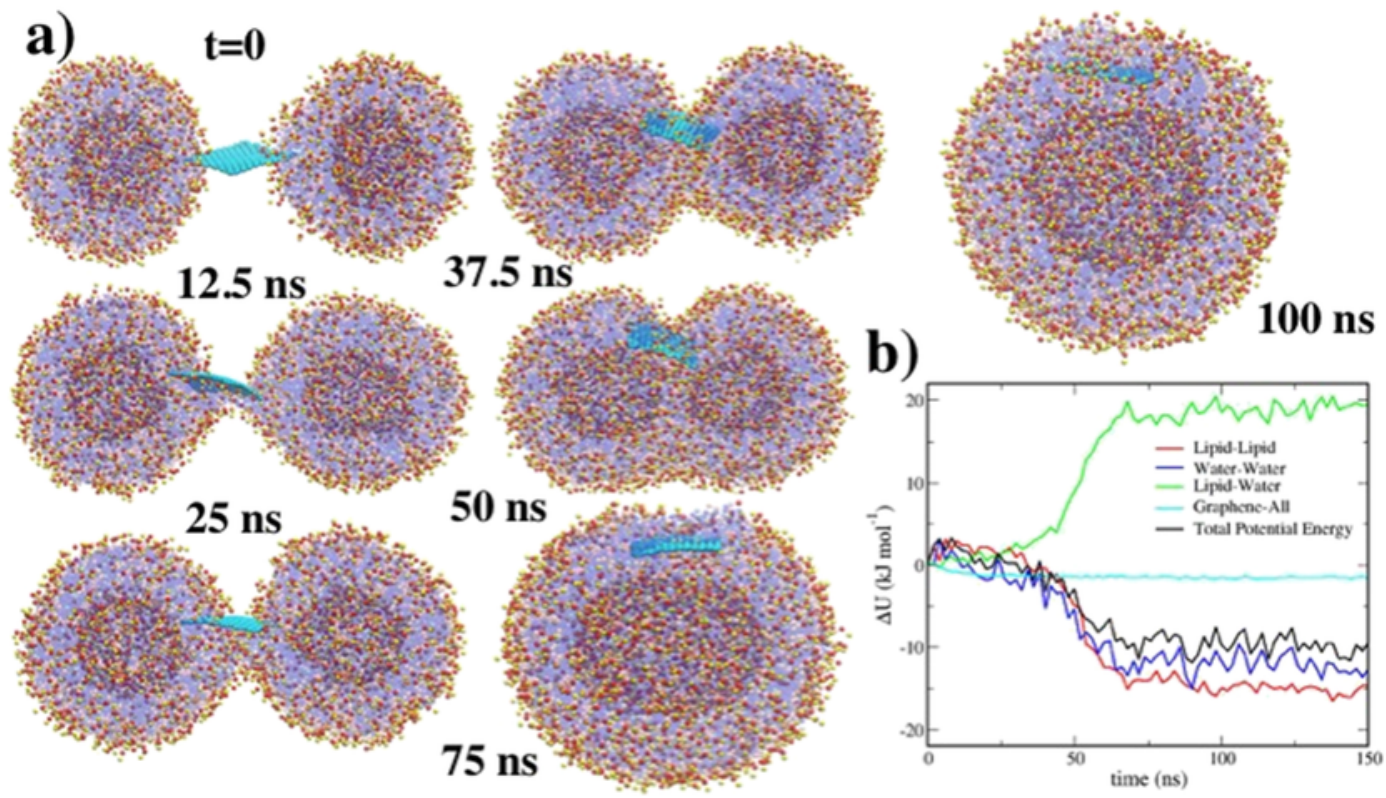

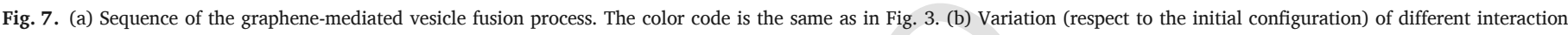
potential contributions, $\Delta \mathrm{U}_{\mathrm{i}-\mathrm{j}}$, during the simulated fusion process. Variation of the total potential interaction energy is also plotted.

graphene size and oxidation degree but also the local curvature of the hosting membrane determines the orientation of embedded graphene particles.

Importantly, we have also reported a clear depletion of cholesterol around inserted graphene layers. An entropic-based rationale has been proposed by suggesting that the rigidity of cholesterol molecules greatly impedes the motion and rotation degrees of freedom of a graphene nanosheet inside the membrane, so it prefers to be surrounded by more flexible and conformable phospholipid molecules.

Finally, our simulations have captured and described spontaneous vesicle fusion when a graphene sheet contacts at the same time with two liposomes. During insertion into the two membranes, graphene is able to perturb and extract lipid molecules from them, facilitating the rupture of the membranes and the reorganization required for the fusion of the two liposome bilayers. The analysis of the energetics of the process has revealed that graphene-mediated vesicle fusion is spontaneous since no activation energy is required for its completion.

From a biological perspective, the interaction modes reported here contribute to a better understanding of the influence of graphene-based materials on lipid bilayers forming biological membranes, in particular in those circumstances where curvature may have a relevant role. So far, all previous works on graphene-membrane interactions are based on simulations of flat bilayers, which are indeed good models to capture most aspects of the general behavior of biological membranes. However, the membranes of biological interest (plasma membranes and also the membranes shaping the internal organelles) are far from flat: they are constantly subjected to changes in their local shape due to dynamic changes of its local composition, the influence of anchored/inserted proteins, the effect of ion channels and pumps, the interaction with the cytoskeleton, and a huge variety of active processes taking place in the membrane $[22,23]$. Actually, the ability of the cell membrane to create and maintain high-curvature compartments is a fundamental requirement for eukaryotic life [22]. The results reported here are then complementary to the findings extracted from planar membranes and they may be applied to biological membranes in those situations/regions where curvature plays an important role. Although our simulations with small liposomes correspond to the limit case of extremely large curvatures (much larger than, for instance, the curvature of a typical plasma membrane), we can learn from them the general trends of the effects produced by membrane curvature on its interaction with graphene particles. Some additional simulations have been performed with the vesicle obtained from the fusion simulation in Fig. 7 (diameter $\sim 15 \mathrm{~nm}$ ). We removed the inserted graphene, we equilibrated the vesicle for 1 microsecond, and we then performed simulations with small and large-sized graphene sheets. These simulations reproduce the main characteristics of the adsorption and insertion modes reported in Figs. 2 and 3, and also the perpendicular/tilted orientation of the inserted nanosheets observed for the small liposome. Therefore, the reported results appear to be consistent for lower curvatures. However, due to computational limitations, a coarse-grained approach for larger vesicles (lower curvatures) is out of reach. The use of molecular coarse-grained approaches with implicit solvent opens new possibilities to explore larger membrane systems while still keeping the molecular details of the lipid components [46].

Although graphene derivatives have broad potential applications in biomedical engineering and biotechnology [5-9], their biological effects are still unclear, and existing studies regarding their biocompatibility frequently present inconclusive results. There is, however, a general consensus provided by both in vitro and in vivo studies that graphene particles display different levels of toxicity depending on the experimental conditions and the properties of the nanomaterial. Some results have indicated that the physicochemical properties such as size, oxidation degree and number of layers, are closely related to the cytotoxicity, and determine the biodistribution of graphene [7]. Studies often show that eukaryotic cell viability decreases after exposure to graphene materials, as they induce oxidative stress and apoptosis [12]. Such cytotoxicity is generally attributed to accumulation of graphene on cell membranes and the consequent physical damage and/or alteration of membrane function [13].

Our simulations report different interaction modes between graphene particles and lipid bilayers at the molecular level. Nanometric graphene layers can be adsorbed and inserted in lipid membranes in different manners, implying important consequences on the structure and functionality of biological cell membranes, and on the bioaccumulation of graphene in living organisms. Moreover, modification of cholesterol distribution in the membrane due to the presence of graphene may also affect many cellular functions where cholesterol distribution is important. How graphene alters membrane structure and lipid orga- 
nization is therefore of key importance to understand its impact on the biological function, and here we offer a molecular perspective of this issue for curved cholesterol-containing bilayers. Finally, the fusogenic ability of graphene nanosheets reported here opens new possibilities for vesicle fusion than deserve to be explored systematically both in the computational and experimental contexts.

\section{Conflicts of interests}

The authors declare no conflict of interest.

\section{Acknowledgements}

The authors acknowledge financial support from the Spanish Ministry of Economy and Competitiveness (MINECO) through project FIS 2013-41144P

\section{References}

[1] C.N.R. Rao, A.K. Sood, K.S. Subrahmanyam, et al., Graphene: the new two-dimen sional nanomaterial, Angew. Chem. Int. Ed. 48 (2009) 7752-7777.

[2] A.K. Geim, K.S. Novoselov, The rise of graphene, Nat. Mater. 6 (2007) 183-191.

[3] F. Piccinno, F. Gottschalk, S. Seeger, B. Nowack, Industrial production quantities and uses of ten engineered nanomaterials in Europe and the world, J. Nanopart. Res. 14 (2012) 1109.

[4] R.H. Hurt, M. Monthioux, A. Kane, Toxicology of carbon nanomaterials: status, trends, and perspectives on this special issue, Carbon 44 (2006) 1028-1033.

[5] C. Chung, Y.-K. Kim, D. Shin, S.-R. Ryo, B.H. Hong, D.-H. Min, Biomedical applications of graphene and graphene oxide, Acc. Chem. Res. 46 (2013) 2211-2224.

[6] H. Shen, L. Zhang, M. Liu, Z. Zhang, Biomedical applications of graphene, Theranostics 2 (2012) 283-294.

[7] L.Z. Feng, Z. Liu, Graphene in biomedicine: opportunities and challenges, Nanomedicine 6 (2) (2011) 317-324.

[8] V. Snitka, Graphene Based Materials: Opportunities and Challenges in Nanomedicine, vol. 2, 201500035

[9] G. Reina, J.M. González-Domínguez, A. Criado, E. Vázquez, A. Bianco, M. Prato, Promises, facts and challenges for graphene in biomedical applications, Chem. Soc Rev. 46 (2017) 4400-4416.

[10] K. Wang, J. Ruan, H. Song, J. Zhang, Y. Wo, S. Guo, D. Cui, Biocompatibility of graphene oxide, Nanoscale Res. Lett. 6 (2011) 8-16.

[11] V.C. Sanchez, A. Jachak, R.H. Hurt, A.B. Kane, Biological interactions of graphene-family nanomaterials: an interdisciplinary review, Chem. Res. Toxicol. 25 (2014) 15-34.

[12] A.M. Pinto, I.C. Goncalvez, F.D. Magalhäesa, Graphene-based materials biocompat ibility: a review, Colloids Surf. B: Biointerfaces 111 (2013) 188-202.

[13] W. Hu, C. Peng, M. Lv, X. Li, Y. Zhang, et al., Protein corona mediated mitigation of cytotoxicity of graphene oxide, ACS Nano 5 (2011) 3693-3700.

[14] R. Frost, S. Svedhem, C. Langhammer, B. Kasemo, Graphene oxide and lipid membranes: size-dependent interactions, Langmuir 32 (2016) 2708-2717.

[15] X. Liu, K.L. Chen, Interactions of graphene oxide with model cell membranes: probing nanoparticle attachment and lipid bilayer disruption, Langmuir 31 (2015) $12076-12086$

[16] P.-J.J. Huang, F. Wang, J. Liu, Liposome/graphene oxide interaction studied by isothermal titration calorimetry, Langmuir 32 (2016) 2458-2463.

[17] R. Zappacosta, M. Di Giulio, V. Ettorre, D. Bosco, C. Hadad, G. Siani, S. Di Bartolomeo, A. Cataldi, L. Cellini, A. Fontana, Liposome-induced exfoliation of graphite to few-layer graphene dispersion with antibacterial activity, J. Mater. Chem. B 3 (2015) 6520-6527.

[18] Y. Li, H. Yuan, A. von dem Bussche, M. Creighton, R.H. Hurt, A.B. Kane, H. Gao, Graphene microsheets enter cells through spontaneous membrane penetration at edge asperities and corner sites, Proc. Natl. Acad. Sci. 110 (2013) 12295-12300.

[19] J. Wang, Y. Wei, X. Shi, H. Gao, Cellular entry of graphene nanosheets: the role of thickness, oxidation and surface adsorption, RCS Adv. 3 (2013) 15776-15782.
[20] A.V. Titov, P. Král, R. Pearson, Sandwiched graphene-membrane superstructures, ACS Nano 4 (2010) 229-234.

[21] N. Willems, A. Urtizberea, A.F. Verre, M. Iliut, M. Lelimousin, M. Hirtz, A. Vijayaraghavan, M.S.P. Sansom, Biomimetic phospholipid membrane organization on graphene and graphene oxide surfaces: a moleculars dynamics simulation study, ACS Nano 11 (2017) 1613-1625.

[22] H.T. McMahon, E. Boucrot, Membrane curvature at a glance, J. Cell Sci. 128 (2015) 1065-1070.

[23] I.K. Jarsch, F. Daste, J.L. Gallop, Membrane curvature in cell biology: an integration of molecular mechanisms, J. Cell Biol. 214 (2016) 375-387.

[24] M.D. Houslay, K.K. Stanley, Dynamics of Biological Membranes: Influence on Synthesis, Structure, and Function, Wiley \& Sons Ed., New York, 1982.

[25] S.J. Marrink, H.J. Risselada, S. Yefimov, D.P. Tieleman, A.H. de Vries, The MARTINI force field: coarse grained model for biomolecular simulations, J. Phys. Chem B 111 (2007) 7812-7824.

[26] S.J. Marrink, D.P. Tieleman, Perspective on the Martini model, Chem. Soc. Rev. 42 (2013) 6801-6822.

[27] J. Sastre, I. Mannelli, R. Reigada, Effects of fullerene on lipid bilayers displaying different liquid ordering: a coarse-grained molecular dynamics study, Biochim. Biophys. Acta Gen. Subj. 1861 (2017) 2872-2882.

[28] L. Ruiz, W. Xia, Z. Meng, S. Keten, A coarse-grained model for the mechanical be havior of multi-layer graphene, Carbon 82 (2015) 103-115.

[29] D. Wu, X. Yang, Coarse-grained molecular simulation of self-assembly for nonionic surfactants on graphene nanostructures, J. Phys. Chem. B 116 (2012) 12048-12056.

[30] E.J. Wallace, M.S.P. Sansom, Carbon nanotube/detergent interactions via coarse-grained molecular dynamics, Nano Lett. 7 (2007) 1923-1928.

[31] J. Wong-Ekkabut, S. Baoukina, W. Triampo, I.-M. Tang, D.P. Tieleman, L. Monticelli, Computer simulation study of fullerene translocation through lipid membranes, Nat. Nanotechnol. 3 (2008) 363-368.

[32] E. Lindahl, B. Hess, D. van der Spoel, GROMACS 3.0: a package for molecular simulation and trajectory analysis, J. Mol. Model. 7 (2001) 306-317.

[33] I. Mannelli, F. Sagués, V. Pruneri, R. Reigada, Lipid vesicle interaction with hydrophobic surfaces: a coarse-grained molecular dynamics study, Langmuir 32 (2016) 12632-12640.

[34] W. Humphrey, A. Dalke, K. Schulten, VMD - Visual Molecular Dynamics, J. Mol. Graph. 14 (1996) 33-38 http://www.ks.uiuc.edu/Research/vmd/.

[35] I. Mannelli, R. Reigada, I. Suárez, D. Janner, A. Carrilero, P. Mazumder, F. Sagués, V. Pruneri, M. Lakadamyali, Functionalized surfaces with tailored wettability determine Influenza a infectivity, ACS Appl. Mater. Interfaces 8 (2016) 15058-15066.

[36] I. Mannelli, D. Janner, F. Sagués, R. Reigada, Assessing the optimal conditions for surface-mediated disinfection of Influenza a virus solutions, Environ. Chem. 14 (2017) 319-326.

[37] L.V. Chernomordik, V.A. Frolov, J. Zimmerberg, Lipids in biological membrane fusion, J. Membr. Biol. 146 (1995) 1-14.

[38] D.P. Siegel, The modified stalk mechanism of lamellar/inverted phase transitions and its implications for membrane fusion, Biophys. J. 76 (1999) 291-313.

[39] S.J. Marrink, A.E. Mark, The mechanism of vesicle fusion as revealed by molecular dynamics simulations, J. Am. Chem. Soc. 125 (2003) 11144-11145.

[40] V. Knecht, S.J. Marrink, Molecular dynamics simulations of lipid vesicle fusion in atomic detail, Biophys. J. 92 (2007) 4254-4261.

[41] L. Gao, R. Lipowsky, J. Shillcock, Tension-induced vesicle fusion: pathways and pore dynamics, Soft Matter 4 (2008) 1208-1214.

[42] P.M. Kasson, E. Lindahl, V.S. Pande, Atomic-resolution simulations predict a transition state for vesicle fusion defined by contact of a few lipid tails, PLoS Comp. Biol. 6 (2010) e1000829.

[43] A. Grafmüller, J. Shillcock, R. Lipowsky, The fusion of membranes and vesicles: pathway and energy barriers from dissipative particle dynamics, Biophys. J. 96 (2009) 2658-2675.

[44] H.J. Risselada, G. Bubnis, H. Grubmüller, Expansion of the fusion stalk and its implication for biological membrane fusion, Proc. Natl. Acad. Sci. U. S. A. 111 (2014) 11043-11048.

[45] Y. Kozolovsy, M. Kozlov, Stalk model of membrane fusion: solution of energy crisis, Biophys. J. 82 (2002) 882-895.

[46] C. Arnarez, et al., Dry Martini, a coarse-grained force field for lipid membrane simulations with implicit solvent, J. Chem. Theor. Comput. 11 (2015) 260. 